\title{
A Numerical Model of Carbon Dioxide Concentration Measurement in a Room with an Opened Ventilation System
}

\author{
Wasu Timpitak ${ }^{1,2}$, Nopparat Pochai ${ }^{1,2,{ }^{*}}$ \\ ${ }^{1}$ Department of Mathematics, King Mongkut's Institute of Technology Ladkrabang, Bangkok 10520, Thailand \\ ${ }^{2}$ Centre of Excellence in Mathematics, CHE, Si Ayutthaya Road, Bangkok 10400, Thailand
}

Received March 5, 2021; Revised June 5, 2021; Accepted June 20, 2021

Cite This Paper in the following Citation Styles

(a): [1] Wasu Timpitak, Nopparat Pochai, "A Numerical Model of Carbon Dioxide Concentration Measurement in a Room with an Opened Ventilation System," Environment and Ecology Research, Vol.9, No.3, pp. 107-113, 2021. DOI: 10.13189/eer.2021.090302

(b): Wasu Timpitak, Nopparat Pochai, (2021). A Numerical Model of Carbon Dioxide Concentration Measurement in a Room with an Opened Ventilation System. Environment and Ecology Research, 9(3), 107-113. DOI: 10.13189/eer.2021.090302

Copyright $@ 2021$ by authors, all rights reserved. Authors agree that this article remains permanently open access under the terms of the Creative Commons Attribution License 4.0 International License

\begin{abstract}
A vast number of patients visit the facility every day, causing a major air pollution issue that may pose a risk of exposure of respiratory infectious diseases in outpatient rooms and harm human health. TB, COVID-19, MERS, and SARS are dangerous communicable diseases that transmit from person to person through the air or aerosol in a variety of forms, such as coughing, spitting, sneezing, speaking, or through wounds. COVID-19, TB, MERS and SARS are risks and the chances of success toward lethal infection make more patients ill in the hospital. We should also be notified of the care and control of these diseases. As a result, effective air quality monitoring is needed to monitor and reduce the potential for infected air, such as carbon dioxide $(\mathrm{CO} 2)$ concentrations. Measuring and controlling carbon dioxide in a hospital with a ventilation system where the number of patients in each room varies in time is challenging. In this research, the numerical model of carbon dioxide concentration measurement in a space with an opened ventilation system is proposed. The model sets the concentration of carbon dioxide at any point when the number of people and the rate of ventilation varies. The classical fourth-order Runge-Kutta method is employed to approximate the model solution. There are many cases of scenarios for improving air quality in the proposed simulations. In the air quality management process, the proposed model provides a balance between the number of persons allowed to stay in the room and the capacity of the air ventilation system.
\end{abstract}

Keywords Airborne, Infectious, Diseases, Ventilation System

\section{Introduction}

In [1],[2],[3],[4], and [5], they proposed that infectious disease of airborne such as tuberculosis (TB) spread in several gathering locate areas with infectors and poor ventilation per person rates, [1],[2],[3],[4], and [5]. In [6],[7],[8], and [4], they proposed infectors could be dangerous if there no is a high concentration of indoor rebreathed air because it could contain infector-borne infectious particles, which could lead to the spread of airborne infectious diseases like tuberculosis. In [6], and [9], they proposed carbon dioxide be used as an indicator of air quality indoor, built on the notion that people release carbon dioxide at a rate dictated by their body weight and bodily movement, and that levels of carbon dioxide indoor are measured by fresh air clearance. In [6],[10], and [9], they propose carbon dioxide concentration in the air of approximately $400 \mathrm{ppm}$ in a room, but when but people enter it, exhaled air concentration begins to rise, depending on the rate of ventilation per person, the length of the room, and the number of persons who are present in the room, because of their oxygen intake, respiratory quotient, and bodily movement, person in the room add to the rise in rebreathed air. In [4], and [2], they proposed that as the exhaled air concentration in a room rises in the presence of infectors, the probability of vulnerable individuals contracting infectious diseases transmitted by the air, this is because contaminated people's exhaled air also contains con- 
tagious airborne particles inside the nuclei with droplets that can stay airborne for extended periods and infect a susceptible person when inhaled. In [2],[11], and [12], they proposed the immune system's condition of the host, host physiology, and the virulence of the Mycobacterium tuberculosis (Mtb) infectious strain are all important factors in the advancement of infection to TB disease.

In [13],they proposed that respiratory activities including talking, coughing, sneezing, and singing may contribute to the formation of respiratory particles. In [14], and [15], they proposed that when a susceptible individual inhales airborne infectious particles, only a proportion of the infectious particles inhaled successfully infiltrate the target region of respiratory tract infection. In [16], they proposed a numerical model that may be used to explain the dynamic dispersion of airborne infectious illnesses in an outpatient room. In [17], and [12], they proposed that infectious particles with a key size range of $1 \mathrm{Mm}$ to $5 \mathrm{Mm}$ had a higher possibility of reaching and depositing on the alveolar area than those with sizes greater than $5 \mathrm{Mm}$, which are confined in the upper respiratory tract. This means that not all infectious particles absorbed from the air will reach or be kept at the site of infection. As a result, while evaluating the risk of airborne infectious illness, the respiratory deposition fraction of airborne infectious particles must be included. In [19], the main route is a droplet or an airborne transmission, the risk of infection is known to be much lower outside where ventilation is better. As winter approaches in the northern hemisphere, opportunities for socialization and outdoor exercise are becoming more challenging and concerns about the increased risk of COVID-19 transmission are growing. In [20], They proposed that about the efficacy of ventilation systems for human thermal comfort in terms of ceiling height, which contributes to green building architectures. Other advantages of ventilation, which we gain in high-ceilinged dwellings, cannot be overlooked. This would also assist to minimize moisture, smoke, odor, heat, dust, and germs. In this research, several numerical models of carbon dioxide concentration measurement in a room with an opened ventilation system is introduced.

\section{The amount of rebreathed air inhaled in the room induce to cause infection.}

In general, the rate of exhaled air generation and ventilation per person determine the raised concentration of indoor carbon dioxide [6],[8], and [9]. Because an infected individual's exhaled air contains airborne infectious particles, carbon dioxide levels can be employed as an exhaled air surrogate [6],[7],[9],[4], and [18]. Exhaled air contains approximately $40,000 \mathrm{ppm}$ of carbon dioxide, compared to $400 \mathrm{ppm}$ of carbon dioxide in ambient air [6],[4], and [3].

We assume that an indoor space, such as a room with a volume of $V$, begins the day with a carbon dioxide concentration of $C_{E}$ of about $400 \mathrm{ppm}$ and is occupied by a number of people, $n$. Given the presence of infectors, the concentration of exhaled air that may contain airborne contagious particles may tend to rise in the room, based on the rate of ventilation, $Q$, and the number of people in the room. We simply assume that persons in the room contribute substantially to the production of carbon dioxide, which serves as an exhaled air marker. The fundamental equation of the accumulation rate exhaled air concentration in a room with carbon dioxide environmental, is equal to the exhaled air rate generated by inhabitants plus the rate of carbon dioxide environmental, minus ventilation rate removes exhaled air:

$$
V \frac{d C}{d t}=n p C_{a}+Q C_{E}-Q C,
$$

where $C$ is the concentration of indoor air exhaled (ppm), $p$ is the rate of breathing $(L / s)$ for each person in the room and $C_{a}$ is the carbon dioxide fraction included in inbreathed air. $t$ is the duration time and $T$ is the stationery simulation time. Initial condition $C(0)=C_{0}$ where $C_{0}$ is the latent carbon dioxide concentration.

If the value of $Q$ assumed by $Q_{i n}$ and $Q_{\text {out }}$, then these values are named the inlet ventilation rate and the outlet ventilation respectively and in a simple scenario, a number of people are unstable then a number of people depend on the time assumed by $n(t)$. In this study preferred to use Eq.(1) as follow:

$$
V \frac{d C}{d t}=n(t) p C_{a}+Q_{i n} C_{E}-Q_{o u t} C,
$$

for all $0 \leq t \leq T$.

\section{Numerical technique}

A continuous approximation to the solution $C(t)$ will not be obtained; instead, approximations to $C$ will be generated at various values, called mush points, in the interval $[0, T]$.

Once the approximate solution is obtained at the points, the approximate solution at other points in the interval can be found by interpolation. We first make the stipulation that the mesh points are equally distributed throughout the interval $[0, T]$. This condition is ensured by choosing a positive integer $N$ and selecting the mesh points $t_{i}=a+i h$, for each $i=0,1,2, \ldots, N$. The common distance between the points $h=(T-0) / N=t_{i+1}-t_{i}$ is called the step size.

\section{1 the classical fourth-order Runge-Kutta method}

$$
\begin{aligned}
C & \cong C_{i} \\
C_{i+1} & =C_{i}+\frac{1}{6}\left(k_{1}+2 k_{2}+2 k 3+k_{4}\right) h \\
k_{1} & =f\left(t_{i}, C_{i}\right) \\
k_{2} & =f\left(t_{i}+\frac{1}{2} h, C_{i}+\frac{1}{2} k_{1} h\right) \\
k_{3} & =f\left(t_{i}+\frac{1}{2} h, C_{i}+\frac{1}{2} k_{2} h\right) \\
k_{4} & =f\left(t_{i}+h, C_{i}+k_{3} h\right)
\end{aligned}
$$


from Eq.(2), we get the classical fourth-order RK method

$$
\begin{aligned}
\frac{d C}{d t} & =f\left(t_{i}, C_{i}\right) \\
f\left(t_{i}, C_{i}\right) & =\frac{1}{V}\left(n(t) p C_{a}+Q_{\text {in }} C_{E}-Q_{o u t} C_{i}\right)
\end{aligned}
$$

\section{Numerical experiments and results}

Assuming that the class room of volume $V=75\left(\mathrm{~m}^{3}\right)$, each person's breathing rate in the room assumed by $p=0.12(\mathrm{~L} / \mathrm{s})$ and the carbon dioxide fraction included in inbreathed air $C_{a}=$ 0.04 ,

\subsection{Simulation 1: an ideal carbon dioxide concentration measurement.}

Table 1 lists the model's physical parameters. $C_{0}=0.01$ is the ambient carbon dioxide concentration (ppm). The analytical solution for this case can be obtained by [21] such as,

$$
C(t)=C_{E}+\frac{n p C_{a}}{Q}\left[1-e^{-Q t / V}\right] .
$$

Table 2 presents the approximated solution's maximum errors. As seen in Fig 1, the approximated solutions are compared to the analytical solution.

Table 1. Physical parameters.

\begin{tabular}{|c|c|c|}
\hline$n(t)$ & $C_{E}$ & $Q$ \\
\hline 50 & 0.004 & 8 \\
\hline
\end{tabular}

Table 2. The maximum $l_{2}$ norm error of the RK4 solution with the analytical solution.

\begin{tabular}{|c|c|}
\hline$\triangle t$ & Maximum error \\
\hline 0.100 & $0.1660 \times 10^{-10}$ \\
0.050 & $0.0146 \times 10^{-10}$ \\
0.025 & $0.0013 \times 10^{-10}$ \\
\hline
\end{tabular}

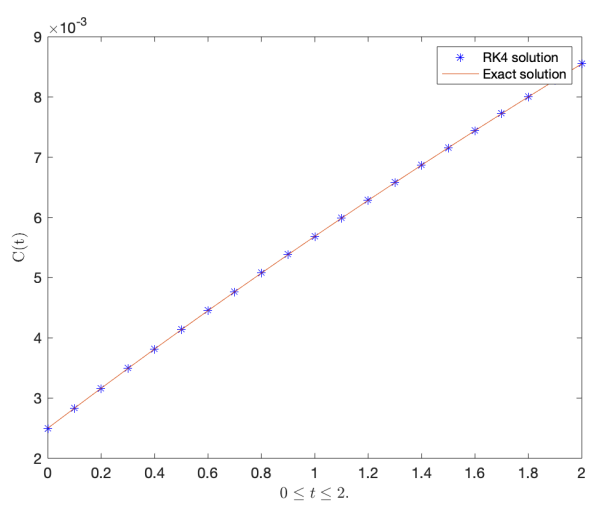

Figure 1. The comparison of the RK4 solution and the analytical solution in a room with a ventilation system $\triangle t=0.1 T=180$.

\subsection{Simulation 2: a small number of people in the room carbon dioxide concentration measurement.}

Table 3 lists the physical parameters. $C_{0}=0.01,0.005$, and 0.0025 are the initial carbon dioxide concentrations. We achieve the approximated solutions illustrated in Fig 2 by using the RK4 method Eqs.(3)-(10).

Table 3. Physical parameters.

\begin{tabular}{|c|c|c|c|}
\hline$n(t)$ & $C_{E}$ & $Q_{\text {in }}$ & $Q_{\text {out }}$ \\
\hline 5 & 0.004 & 8 & 4 \\
\hline
\end{tabular}

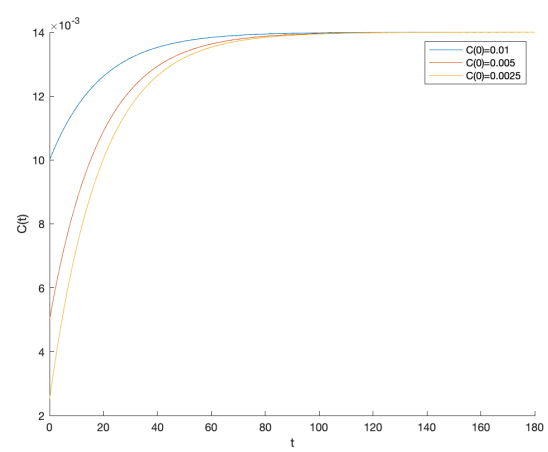

Figure 2. The approximated carbon dioxide concentration in a room with a ventilation system $\triangle t=0.1 T=180$.

\subsection{Simulation 3: a large number of people in the room carbon dioxide concentration measurement.}

Table 4 lists the physical parameters. $C_{0}=0.01,0.005$, and 0.0025 are the initial carbon dioxide concentrations. We achieve the approximated solutions illustrated in Fig 3 by using the RK4 method Eqs.(3)-(10).

Table 4. Physical parameters.

\begin{tabular}{|c|c|c|c|}
\hline$n(t)$ & $C_{E}$ & $Q_{\text {in }}$ & $Q_{\text {out }}$ \\
\hline 50 & 0.004 & 8 & 4 \\
\hline
\end{tabular}

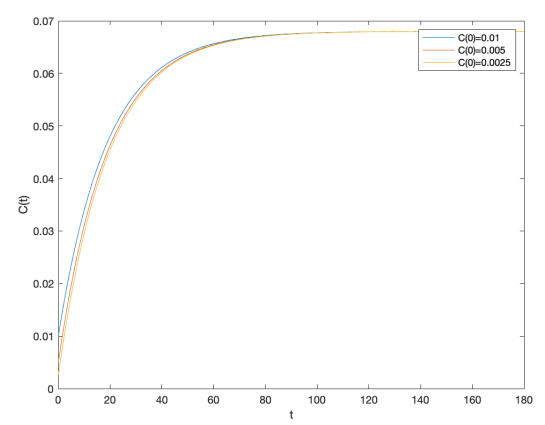

Figure 3. The approximated carbon dioxide concentration in a room with a ventilation system $\triangle t=0.1 T=180$. 


\subsection{Simulation 4: a different number of people in the room carbon dioxide concentration measurement.}

Table 5 lists the physical parameters. $n(t)=5,25$ and 50 are a number of people. We achieve the approximated solutions illustrated in Figs 4-6 by using the RK4 method Eqs.(3)-(10).

Table 5. Physical parameters.

\begin{tabular}{|c|c|c|c|}
\hline$C_{0}$ & $C_{E}$ & $Q_{\text {in }}$ & $Q_{\text {out }}$ \\
\hline 0.01 & 0.004 & 4 & 8 \\
\hline
\end{tabular}

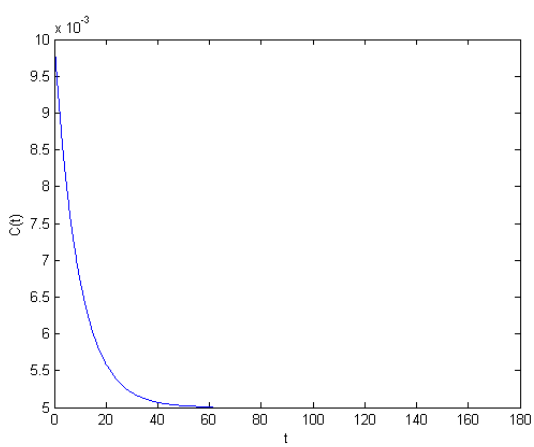

Figure 4. The approximated carbon dioxide concentration in a room with a ventilation system $\triangle t=0.1 T=180$.

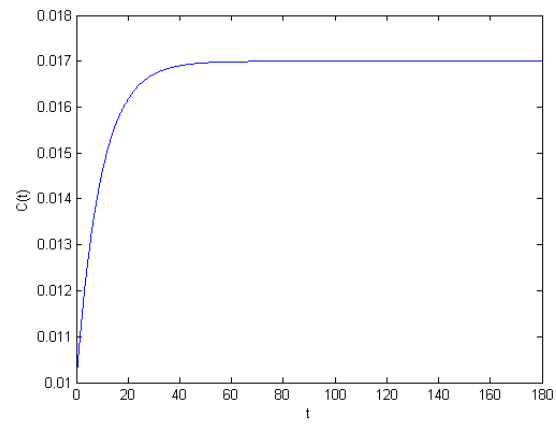

Figure 5. The approximated carbon dioxide concentration in a room with a ventilation system $\triangle t=0.1 T=180$.

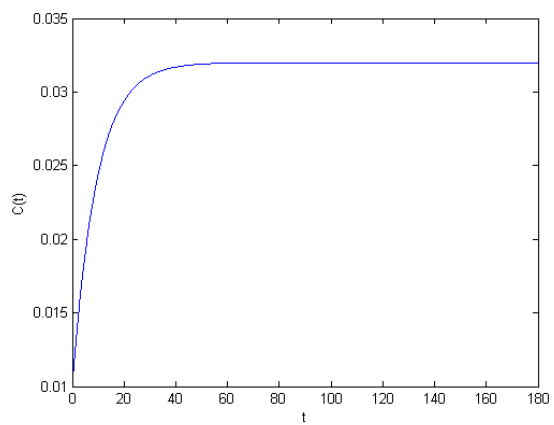

Figure 6. The approximated carbon dioxide concentration in a room with a ventilation system $\triangle t=0.1 T=180$.

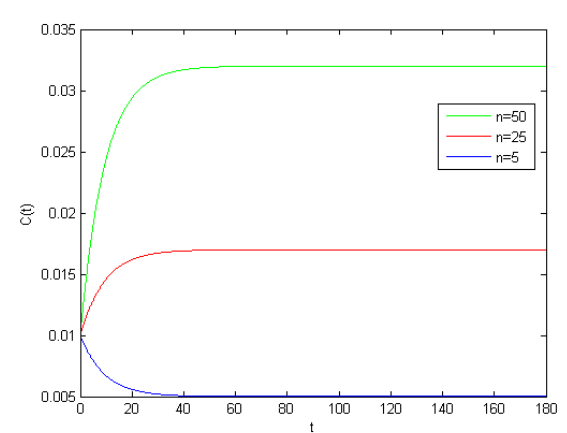

Figure 7. The comparison of RK4 solutions.

\subsection{Simulation 5: varying number of people in the room carbon dioxide concentration measurement.}

Table 6 lists the physical parameters. A number of people are unstable as show in table 7 . We achieve the approximated solutions illustrated in Fig 8 by using the RK4 method Eqs.(3)(10).

Table 6. Physical parameters.

\begin{tabular}{|c|c|c|c|}
\hline$C_{0}$ & $C_{E}$ & $Q_{\text {in }}$ & $Q_{\text {out }}$ \\
\hline 0.0025 & 0.004 & 8 & 4 \\
\hline
\end{tabular}

Table 7. A number of people $n(t)$.

\begin{tabular}{|c|c|c|c|c|c|c|c|c|c|c|}
\hline$t$ & 0 & 20 & 40 & 60 & 80 & 100 & 120 & 140 & 160 & 180 \\
\hline$n(t)$ & 5 & 10 & 15 & 30 & 45 & 50 & 45 & 30 & 20 & 10 \\
\hline
\end{tabular}

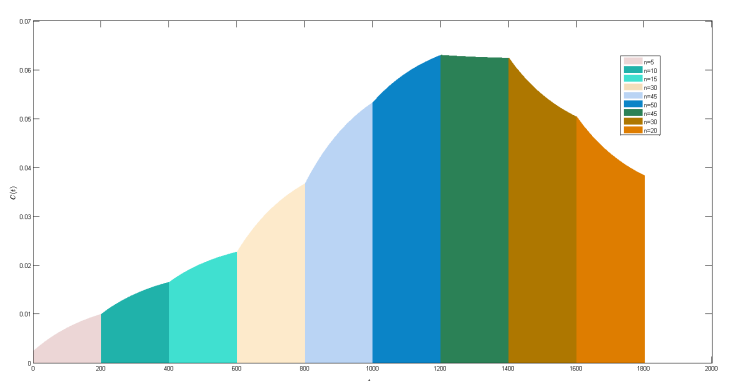

Figure 8. The approximated carbon dioxide concentration in a room with a ventilation system when the number of people are unstable $\Delta t=0.1 T=$ 180 .

\subsection{Simulation 6: changing rate of ventilation in a room carbon dioxide concentration measurement.}

Table 8 lists the physical parameters. The rate of ventilations are unstable as show in table 9. We achieve the approximated solutions illustrated in Fig 9 by using the RK4 method Eqs.(3)(10). 
Table 8. Physical parameters.

\begin{tabular}{|c|c|c|}
\hline$C_{0}$ & $C_{E}$ & $n(t)$ \\
\hline 0.0025 & 0.004 & 50 \\
\hline
\end{tabular}

Table 9. The rate of ventilations.

\begin{tabular}{|c|c|c|c|}
\hline$t$ & $0-60$ & $61-140$ & $141-180$ \\
\hline$Q_{\text {in }}(t)$ & 8 & 4 & 8 \\
$Q_{\text {out }}(t)$ & 4 & 8 & 4 \\
\hline
\end{tabular}

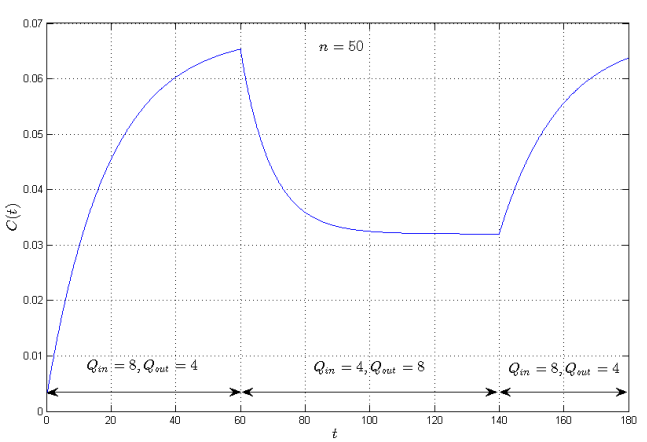

Figure 9. The approximated carbon dioxide concentration in a room with a ventilation system when $n(t)$ is unstable.

\subsection{Simulation 7: the varying number of people when} the outlet ventilation rate greater than inlet ventilation rate in the room carbon dioxide concentration measurement

Table 10 lists the physical parameters. A number of people are unstable as show in table 11 . We achieve the approximated solutions illustrated in Fig 10 by using the RK4 method Eqs.(3)-(10).

Table 10. Physical parameters.

\begin{tabular}{|c|c|c|c|}
\hline$C_{0}$ & $C_{E}$ & $Q_{\text {in }}$ & $Q_{\text {out }}$ \\
\hline 0.0025 & 0.004 & 4 & 8 \\
\hline
\end{tabular}

Table 11. A number of people $n(t)$.

\begin{tabular}{|c|c|c|c|c|c|c|c|c|c|c|}
\hline$t$ & 0 & 20 & 40 & 60 & 80 & 100 & 120 & 140 & 160 & 180 \\
\hline$n(t)$ & 5 & 10 & 15 & 30 & 45 & 50 & 45 & 30 & 20 & 10 \\
\hline
\end{tabular}

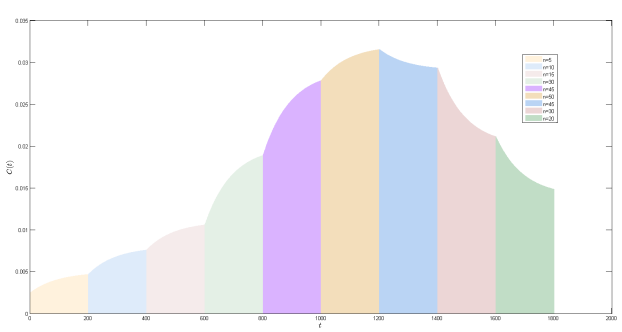

Figure 10. The approximated carbon dioxide concentration in a room with a ventilation system when $n(t)$ is unstable.

\subsection{Simulation 8: changing rate of ventilation which de- pends on the number of people in the room carbon dioxide concentration measurement.}

Table 12 lists the physical parameters. A number of people and the rate of ventilations are unstable as show in table 12-14. We achieve the approximated solutions illustrated in Fig 11 by using the RK4 method Eqs.(3)-(10).

Table 12. Physical parameters.

\begin{tabular}{|c|c|}
\hline$C_{0}$ & $C_{E}$ \\
\hline 0.0025 & 0.004 \\
\hline
\end{tabular}

Table 13. A number of people $n(t)$.

\begin{tabular}{|c|c|c|c|c|c|c|c|c|c|c|}
\hline$t$ & 0 & 20 & 40 & 60 & 80 & 100 & 120 & 140 & 160 & 180 \\
\hline$n(t)$ & 5 & 10 & 15 & 30 & 45 & 50 & 45 & 30 & 20 & 10 \\
\hline
\end{tabular}

Table 14. The rate of ventilations.

\begin{tabular}{|c|c|c|c|}
\hline$t$ & $0-60$ & $61-140$ & $141-180$ \\
\hline$Q_{\text {in }}(t)$ & 8 & 4 & 8 \\
$Q_{\text {out }}(t)$ & 4 & 8 & 4 \\
\hline
\end{tabular}




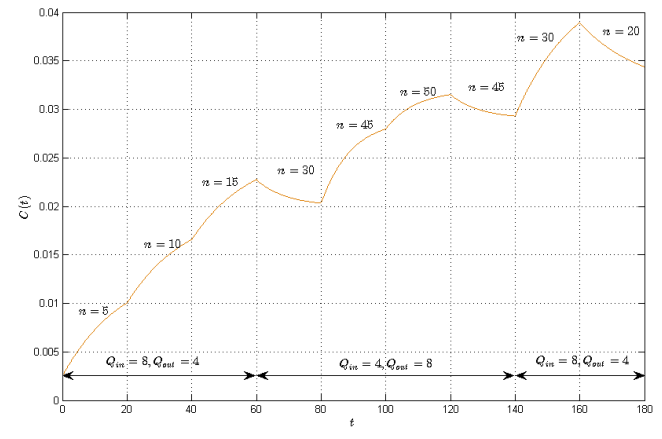

Figure 11. The approximated carbon dioxide concentration in a room with a ventilation system when $n(t), Q_{\text {in }}$ and $Q_{\text {out }}$ are unstable.

\subsection{Simulation 9: number of people is represented as a function in the room carbon dioxide concentration measurement.}

Table 15 lists the physical parameters. A number of people assumed by $n(t)=20+5 \sin (\pi t)$ as show in Fig 12. We achieve the approximated solutions illustrated in Fig 13 by using the RK4 method Eqs.(3)-(10).

Table 15. Physical parameters.

\begin{tabular}{|c|c|c|c|}
\hline$C_{0}$ & $C_{E}$ & $Q_{\text {in }}$ & $Q_{\text {out }}$ \\
\hline 0.0025 & 0.004 & 8 & 4 \\
\hline
\end{tabular}

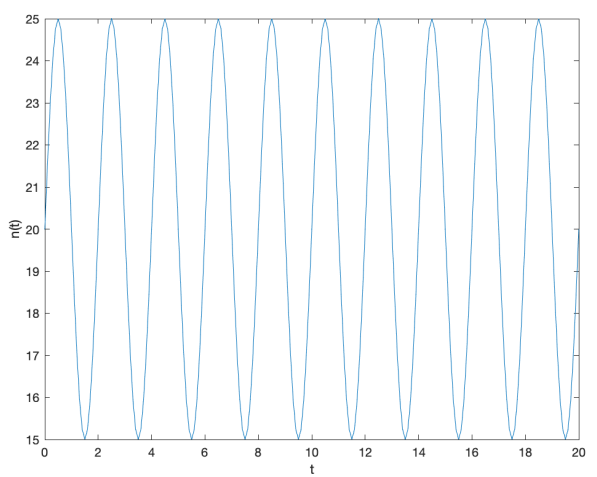

Figure 12. A number of people in a room $0 \leq n(t) \leq 20$.

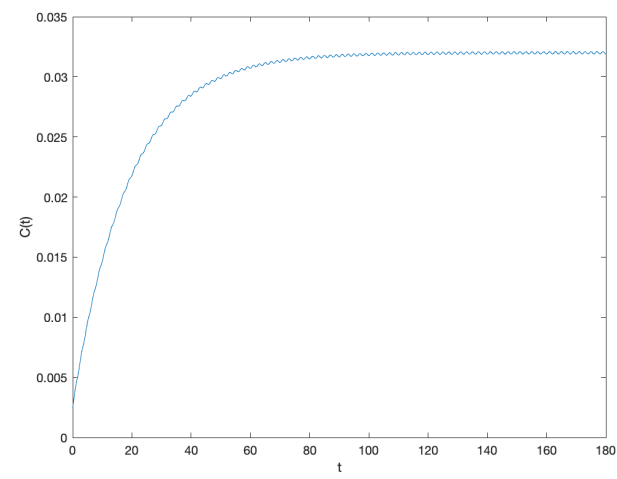

Figure 13. The approximated carbon dioxide concentration in a room with a ventilation system when $n(t)=20+5 \sin (\pi t)$.

\section{Discussion}

In simulation 1, the RK4 solution and the analytical solution is used Eq.(1), The comparison of approximation techniques is illustrated in Fig 3. The RK4 method gives accurately approximated carbon dioxide concentration as show in Table 2.

In simulation 2, we can see that the carbon dioxide concentration along with the starting and the middle of the simulation depends on the potential concentration level. The carbon dioxide concentration for each case becomes close to 0.014 around 1.5 hours, the approximate RK4 solutions when $C(0)$ is divided by a half for each case and assume the number of people $n(t)=5$ as show in Fig 2 .

In simulation 3, we can see that the carbon dioxide concentration along with the starting and the middle of the simulation depends on the potential concentration level and the number of people. When the number of people increases the carbon dioxide concentration is increases. The carbon dioxide concentration for each case becomes close to 0.065 around 1.5 hours, the approximate RK4 solutions when $C(0)$ is divided by a half for each case and assume the number of people $n(t)=50$ as show in Fig 3.

In simulation 4 , when the inlet ventilation rate less than the outlet ventilation rate, the carbon dioxide concentration is reduced at $n(t)=5$, in case $n(t)=25$ and 50 , the carbon dioxide concentration is increases. The carbon dioxide concentration for case 1 becomes close to 0.005 around 1 hours, The carbon dioxide concentration for case 2 becomes close to 0.017 around 1 hours, and The carbon dioxide concentration for case 3 becomes close to 0.033 around 1 hours. The approximate RK4 solutions when assume $n(t)=5,25$ and 50 in 3 case and the inlet ventilation rate less than the outlet ventilation as show in Figs 4-6 and the comparison of 3 cases are illustrated in Fig 7.

In simulation 5, when the number of people is varied, the carbon dioxide concentration of interval $0-2$ hours are increases, we can see that the maximum carbon dioxide concentration is 0.062 , and the carbon dioxide concentration interval 2-3 hours is reduced, the carbon dioxide concentration in last time is 0.04 . The approximate RK4 solutions when $n(t)$ is varied as shown in Fig 8 and the parameter of $n(t)$ as show in 
Table 6.

In simulation 6 , we can see that the carbon dioxide concentration is increases interval $0-1$ hours and $2.3-3$ hours, when the inlet ventilation rate more than the outlet ventilation rate, but the carbon dioxide concentration reduces interval $1-2.3$ hours when the inlet ventilation rate less than the outlet ventilation rate. The approximate RK4 solutions when the rate of ventilations are unstable as shown in Fig 9 and the parameter of the rate of ventilation as shown in Table 9.

In simulation 7 , when the number of people is varied with the inlet ventilation rate less than the outlet ventilation, the carbon dioxide concentration around interval 0-2 hours are increases, we can see that the maximum carbon dioxide concentration is 0.032 , and the carbon dioxide concentration around interval 2-3 hours reduces, the carbon dioxide concentration in last time is 0.015 . the approximate RK4 solutions when $n(t)$ is varied and the inlet ventilation rate less than the outlet ventilations show in Fig 10 and the parameter of $n(t)$ as show in Table 11.

In simulation 8 , the rate of ventilation and the number of people are varied. If the inlet ventilation rate is higher than the outlet ventilation rate, the carbon dioxide concentration will rise. However if the number of people is reduced, the concentration of carbon dioxide is also reduced. In the other hand, if the inlet ventilation rate is smaller than the outlet ventilation rate, the carbon dioxide concentration would decrease. On the other hand, if the inlet ventilation rate is less than the outlet ventilation rate, then the carbon dioxide concentration becomes reducing. However, if the number of people is increased, then the carbon dioxide concentration is also increasing. The approximated RK4 solutions of the simulation are shown in Fig 11 when their parameters are given in Tables 12-14 respectively.

In simulation 9, assume the number of people $n(t)=20+$ $5 \sin (\pi t)$, we can see that the carbon dioxide concentration depends on the number of people. The approximate RK4 solutions when $n(t)=20+5 \sin (\pi t)$ as show in Fig 13 and the number of people in the room as show in Fig 12.

\section{Conclusion}

A computational model is used in this article to estimate the carbon dioxide concentration in a room with an open ventilation system. Because the number of persons in the room varies, a realistic carbon dioxide concentration measurement model is proposed. We can see that the concentration of carbon dioxide depends on the actual concentration level, the number of persons, and the rate of ventilation. We demonstrate that the proposed technique is applicable to real-world problems using the standard fourth order RK method. In an ideal scenario, the approximated solutions are compared to the analytical solution. It was determined that the numerical model produces good agreement findings. The proposed model in the air quality management process achieves a balance between the number of people allowed to remain in the room and the potential of the air ventilation system.

\section{Acknowledgment}

This paper is supported by the Centre of Excellence in Mathematics, the Commission on Higher Education, Thailand. The author greatly appreciates valuable comments received fro the referees.

\section{REFERENCES}

[1] J.R. Andrews, C. Morrow and R. Wood. Modeling the role of public transportation in sustaining tuberculosis transmission in South Africa Am, Vol.177, No.6, 556-561, 2012.

[2] W.F. Well, Airborne Contagion and Air Hygiene. An Ecological Study of Droplet infections, 1995.

[3] S.N. Rudnick, and D.K. Milton. Rick of indoor airborne infection transmission estimated from carbon dioxide concentration, Indoor Air, Vol.13, No.3, 237-245, 2003.

[4] E.T. Richardson, C.D. Morrow, D.B. Kalil, and L.G. Bekker. Shared air: a renewed focus on ventilation for the prevention of tuberculosis transmission, PloS One 9, No.5, e96334, 2014.

[5] L. Gammaitioni, and M.C. Nucci. Using a mathematical model to evaluate the efficacy of TB control measures, Emerg. Infect. Dis, N0.3, 335-342, 1997.

[6] S.J. Emmerich, and A.K. Persily, State-Of-The-Art Review of Carbon Dioxide Bemand Controlled Ventilation Technology an Application, NISTIR 6729, 2001.

[7] Y. Li, G.M. Leung, J.W. Tang, X. Yang, C.Y.H. Chao, J.Z. Lin, and P.L. Yuen. Role of ventilation in airborne transmission of infectious agents in the built environmental multidisciplinary systematic review, Am. Rev. Respir. Dis, Vol.95, No.3, 435442, 2007.

[8] M. Murray, O. Oxlade, and H.H. Lin. Modeling social, environmental and biological determinants of tuberculosis, Int. J. Tuberc. Lsang Dis, Vol.15, No.6, S60-S70, 2011.

[9] A.K. Persily. Evaluating building IAQ and ventilation with indoor carbon dioxide, Trans. Am. Soc. Heat. Refrig. Air. Cond. Eng, N0.103, 193-204, 1997.

[10] M. Lygizos, S.V. Shenoi, B.P. Brooks, A. Bhushan, J.C. Brust, D. Zeltenman, and G.H. Friedland. Natural ventilation reduces high tb transmission risk in traditional homes in rural Kwazulu-Natal, South Africa. BMC Infect. Dis, Vol.13, No.1, $300,2013$.

[11] H.L. Rieder. Socialization pattens are key to the transmission dynamics of tuberculosis, Int. J. Tuberc. Lung Dis, Vol.3, No.3, 177-178, 1999a. 
[12] H.L. Rieder. Epidemiological Basis of Tuberculosis Control (No. Ed. 1), 1-162, 1999b.

[13] R.G. London, and R.M. Roberts. Droplet expulsion from the respiratory tract, Indoor Air, Vol.17, No.1, 2-18, 1967.

[14] C.B. Beggs, C.J. Noakes, P.A. Sleigh , L.A. Fletcher, and K. Siddiqi. the transmission of tuberculosis in confined spaces: an analytical review of alternative epidemiological models, Int. J. Tuberc. Lung Dis, Vol.7, No.11, 1015-1026, 2003.

[15] G.N. Sze To, and C.Y.H. Chao. Review and comparison between the wells-Riley and dose-response approaches to risk assessment of infections respiratory diseases, Indoor Air, Vol.20, No.1, 2-16, 2010.

[16] K. Suebyat, P. Oyjinda, S.A. Konglok, and N. Pochai. A mathematical model for the risk analysis of airborne Infectious disease in an Outpatient room with personal classification factor, IAENG, V01.28, No.4, 2020.
[17] C.J. Noakes, and P.A. Sleigh. Mathematical models for assessing the role of airflow on the risk of airborne infection in hospital wards, J. R. Soc. Interface, Vol.6, No.6, S791-S800, 2009.

[18] R. Wood, C. Morrow, S. Ginsberg, E. Piccoli, D. Kalil, A. Sassi, and J.R. Andrews. Quantification of shared air: a social and environmental determinant of airborne disease transmission, PloS One 9, Vol.9, e106622, 2014.

[19] “COVID-19 transmission up in the air" Lancet Infect Dis 2020, late ed., September 2020, vol 8, issue 12. Editorial.

[20] S. Farooq, F. Zubair and M.A. Evaluation of ventilation system efficiency with reference to ceiling height in warm-humid climate of pakistan, Civil Engineering and Architecture Vol. 8(5), pp. 824 - 831, 2020.

[21] Chacha, M.I. Nicola, M. Robin, W. Modelling the risk of airborne infectious disease using exhaled air, Journal of Theoretical Biology, Vol 372, pp. 100-106, 2015. 